\title{
Radiation Effect on Mhd Heat and Mass Transfer Flow over a Shrinking Sheet with Mass Suction
}

\author{
P. R. Babu ${ }^{1 \dagger}$, J. A. Rao ${ }^{2}$ and S. Sheri ${ }^{3}$ \\ ${ }^{1}$ Dept. of Mathematics, Sri Kottam Tulasi Reddy Memorial College of Engineering, Kondair(village), \\ Itikyala(Mandal), Mahabubnagar(Dist), A.P., India. \\ ${ }^{2}$ Dept. of Mathematics, OsmaniaUniversity, Hyderabad, A.P., India. \\ ${ }^{3}$ Dept. of Mathematics, Githam University, Hyd, A.P., India. \\ †Corresponding Author Email: ramesh.patkar@yahoo.in
}

(Received August 8, 2013; accepted December 22, 2013)

\begin{abstract}
A numerical analysis has been carried out to study the effects radiation and heat source/sink on the steady two dimensional magnetohydrodynamic (MHD) boundary layer flow of heat and mass transfer past a shrinking sheet with wall mass suction. In the dynamic system, a uniform magnetic field acts normal to the plane of flow. The governing partial differential equations are transformed into self-similar equations are solved by employing finite difference using the quasilinearization technique. From the analysis it is found that the velocity inside the boundary layer increases with increase of wall mass suction and magnetic field and accordingly the thickness of the momentum boundary layer decreases. The temperature decreases with Hartmann number, Prandtl number, and heat sink parameter and the temperature increases with heat source parameter, radiation parameter. The concentration decreases with an increase of Hartmann number, mass suction parameter, Schmidt number, chemical reaction parameter.
\end{abstract}

Keywords: Heat and mass transfer, Radiation, MHD, Shrinking sheet, Heat source or sink, Mass suction

\section{INTRODUCTION}

The study boundary layer flow of an incompressible viscous fluid over a stretching sheet has many applications manufacturing industries and technological processes, such as, glass fiber production, wire drawing, paper production, metal and polymer processing industries and many others. Crane (1970) first considered the steady laminar boundary layer flow of a Newtonian fluid caused by a linearly stretching flat sheet and found an exact similarity solution in closed analytical form. Wang (1984) investigated the three-dimensional flow due to the stretching surface.

The MHD boundary layer theory has a significant contribution in developing of magnetohydrodynamic theory. Due to the important effects of magnetic field on the boundary layer, the study of MHD flow with heat transfer is always interesting. In author's knowledge, the first attempt to study the MHD flow over a stretching surface in an electrically conducting fluid was made by Pavlov (1974) in presence of uniform transverse magnetic field and he obtained an exact similarity solution. Furthermore, some important contributions in stretching sheet flow were made by Chakrabarti and Gupta (1979).
Recently, the flow of incompressible fluid due to a shrinking sheet is gaining attention of modern day researchers because of its increasing application to many engineering problems. A steady flow over a shrinking sheet is not possible because the generated vortices are not confined within the boundary layer. So, to overcome this difficultly the flow needs a certain amount of external opposite force at the sheet. Wang (1990) observed the flow around the shrinking sheet while studying the flow behavior of liquid film over an unsteady stretching sheet. Hayat at al. (2007) reported an analytical solution of MHD flow of a second grade fluid over a shrinking sheet. Fang and Zhang (2009) obtained a closed form analytical solution for steady MHD flow over a porous shrinking sheet subject to mass suction. Wang's (2008) studied the stagnation-point flow towards a shrinking sheet.

The study of heat transfer in hydrodynamic boundary layer flow over a porous stretching sheet become more interesting when internal heat generation/absorption occurs. Effects of heat source or sink on the boundary layer flow over a stretching sheet were studied by Bataller (2007), Layek et al. (2007), Chen (2009).

Coupled heat and mass transfer finds applications in a variety of engineering applications, such as the 
migration of moisture through the air contained in fibrous insulation and grain storage installations, filtration, chemical catalytic reactors and processes, spreading of chemical pollutants in plants and diffusion of medicine in blood veins. Free convection flow of an incompressible viscous fluid past an infinite or semi-infinite vertical plate has been studied since long because of its technological importance. Callahan and Marner (1976) solved the problem of transient free convection with mass transfer on an isothermal vertical plate using an explicit finite difference scheme. Unsteady free convective flow on taking into account the mass transfer phenomenon past an infinite vertical porous plate with constant suction was studied by Soundalgekar and Wavre (1977). Soundalgekar (1979) studied the effects of mass transfer and free convection currents on the flow past an impulsively started vertical plate. In these studies the magnetohydrodynamic phenomena is ignored. However in metallurgical transport systems, by drawing plates in an electrically conducting fluid subjected to a transverse magnetic field, the rate of cooling can be controlled and the final desired characteristics can be further refined. Magnetohydrodynamic flow has applications in meteorology, solar physics, cosmic fluid dynamics, astrophysics, geophysics and in the motion of earth's core. Shanker and Kishan (1997) presented the effect of mass transfer on the MHD flow past an impulsively started infinite vertical plate. Elabashbeshy (1997) studied heat and mass transfer along a vertical plate in the presence of magnetic field. Gangadhar and Bhaskar Reddy (2013) analyzed by chemically reacting MHD boundary layer flow of heat and mass transfer over a moving vertical plate in a porous medium with suction.

In the context of space technology and in the processes involving high temperatures, the effects of radiation are of vital importance. Recent developments in hypersonic flights in the paper by Di Clemente et al. (2013), missile re-entry, rocket combustion chambers, power plants for inter planetary flight and gas cooled nuclear reactors, have focused attention on thermal radiation as a mode of energy transfer, and emphasized the need for improved understanding of radiative transfer in these processes. The interaction of radiation with laminar free convection heat transfer from a vertical plate was investigated by Cess (1966) for an absorbing, emitting fluid in the optically thick region, using the singular perturbation technique. Arpaci (1968) considered a similar problem in both the optically thin and optically thick regions and used the approximate integral technique and firstorder profiles to solve the energy equation. Raptis (1998) analyzed the thermal radiation and free convection flow through a porous medium bounded by a vertical infinite porous plate by using a regular perturbation technique. Hossain and Takhar (1996) studied the radiation effects on mixed convection along a vertical plate with uniform surface temperature using the Keller Box finite difference method. In all these papers, the flow is considered to be steady. The unsteady flow past a moving plate in the presence of free convection and radiation were presented by Mansour (1990). Radiation and mass transfer effects on two-dimensional flow past an impulsively started isothermal vertical plate were analyzed by Ramachandra Prasad et al. (2007).

The aim of the present paper is to analyze the steady magnetohydrodynamic (MHD) boundary layer flow due to an exponentially stretching sheet with radiation in the presence of mass transfer and heat source or sink. The governing boundary layer equations have been transformed to a two-point boundary value problem in similarity variables and the resultant problem is solved numerically using the finite difference using the quasilinearization technique. The effects of various governing parameters on the fluid velocity, temperature, concentration, and Nusselt number are shown in figures and analyzed in detail.

\section{MATHEMATICAL FORMULATION}

Consider a steady two dimensional MHD boundary layer flow of an electrically conducting Newtonian and radiating fluid past a shrinking sheet with internal heat generation or absorption. The sheet coincides with the plane $\mathrm{y}=0$ and the flow is confined in the region $y>0$. The $x$ and $y$ axes are taken along and perpendicular to the sheet, respectively. The level of concentration of foreign mass is assumed to be low, so that the Soret and Dufour effects are negligible. Under these assumptions along with the Boussinesq and boundary layer approximations, the system of equations, which models the flow is given by

Continuity equation

$$
\frac{\partial u}{\partial x}+\frac{\partial v}{\partial y}=0
$$

Linear momentum equation

$u \frac{\partial u}{\partial x}+v \frac{\partial u}{\partial y}=v \frac{\partial^{2} u}{\partial y^{2}}-\frac{\sigma B^{2}}{\rho} u$

Energy equation

$u \frac{\partial T}{\partial x}+v \frac{\partial T}{\partial y}=\frac{k}{\rho c_{p}} \frac{\partial^{2} T}{\partial y^{2}}-\frac{1}{\rho c_{p}} \frac{\partial q_{r}}{\partial y}+\frac{Q_{0}}{\rho c_{p}}\left(T-T_{\infty}\right)$

Species equation

$u \frac{\partial C}{\partial x}+v \frac{\partial C}{\partial y}=D \frac{\partial^{2} C}{\partial y^{2}}-k_{0}^{2}\left(C-C_{\infty}\right)$

The boundary conditions for the velocity, temperature and concentration fields are $u=U_{w}(x)=-c x, v=-v_{w}, T=T_{w}, C=C_{w}$

at $y=0$

$u \rightarrow 0, T \rightarrow T_{\infty}, C \rightarrow C_{\infty}$ as $y \rightarrow \infty$

where $u$ and $v$ are the velocity components along the $x$ and $y$ axes, respectively, $\mathrm{c}>0$ is the shrinking constant, $v_{w}(>0)$ is a prescribed distribution of wall mass suction through the sheet, $v=(\mu / \rho)$ is the kinematic fluid viscosity, $\rho$ is the fluid density, $c_{p}$ is the specific heat, $\mathrm{k}$ is the fluid thermal conductivity, $\boldsymbol{B}_{\mathrm{O}}$ is the applied uniform 
magnetic field, $q_{r}$ is the radiative heat flux, $T$ is the temperature, $T_{\infty}$ is the free stream temperature, $C$ is the concentration, $C_{\infty}$ is the free stream concentration, $T_{W}$ is the temperature of the sheet, $C_{w}$ is the concentration of the sheet, $Q_{0}$ is the volumetric rate of heat generation or absorption, $D$ is the coefficient of mass diffusivity. Further, $k_{0}$ is respectively the chemical reaction rate constant.

By using the Rosseland approximation (Brewster (1992)), the radiative heat flux $q_{r}$ is given by

$q_{r}=-\frac{4 \sigma^{*}}{3 K^{\prime}} \frac{\partial T^{4}}{\partial y}$

where $\sigma^{*}$ is the Stefan-Boltzmann constant and $K^{\prime}$ - the mean absorption coefficient. It should be noted that by using the Rosseland approximation, the present analysis is limited to optically thick fluids. If temperature differences within the flow are sufficiently small, then $\mathrm{Eq}$.(2.7) can be linearized by expanding $T^{4}$ into the Taylor series about $T_{\infty}$, which after neglecting higher order terms takes the form

$T^{4} \cong 4 T_{\infty}^{3} T-3 T_{\infty}^{4}$

In view of Eqs. (2.6) and (2.7), Eq.(2.3) reduces to

$u \frac{\partial T}{\partial x}+v \frac{\partial T}{\partial y}=\left(\frac{k}{\rho c_{p}}+\frac{16 \sigma^{*} T_{\infty}^{3}}{3 \rho c_{p} K^{\prime}}\right) \frac{\partial^{2} T}{\partial y^{2}}+\frac{Q_{0}}{\rho c_{p}}\left(T-T_{\infty}\right)$

The continuity Eq. (2.1) is satisfied by the Cauchy Riemann equations

$u=\frac{\partial \psi}{\partial y}$ and $v=-\frac{\partial \psi}{\partial x}$

where $\psi(x, y)$ is the stream function.

For relation of (2.9), the mass-conservation Eq.(1) is satisfied automatically and the Eqs. (2.2), (2.8) and (2.4) take the following forms

$$
\begin{aligned}
& \frac{\partial \psi}{\partial y} \frac{\partial^{2} \psi}{\partial x \partial y}-\frac{\partial \psi}{\partial x} \frac{\partial^{2} \psi}{\partial y^{2}}=v \frac{\partial^{3} \psi}{\partial y^{3}}-\frac{\sigma B_{0}^{2}}{\rho} \frac{\partial \psi}{\partial y}(2.10) \\
& \frac{\partial \psi}{\partial y} \frac{\partial T}{\partial x}-\frac{\partial \psi}{\partial x} \frac{\partial T}{\partial y}=\left(\frac{k}{\rho c_{p}}+\frac{16 \sigma^{*} T_{\infty}^{3}}{3 \rho c_{p} K^{\prime}}\right) \frac{\partial^{2} T}{\partial y^{2}}+\frac{Q_{0}}{\rho c_{p}}\left(T-T_{\infty}\right) \\
& \frac{\partial \psi}{\partial y} \frac{\partial C}{\partial x}-\frac{\partial \psi}{\partial x} \frac{\partial C}{\partial y}=D \frac{\partial^{2} C}{\partial y^{2}}-k_{0}^{2}\left(C-C_{\infty}\right)
\end{aligned}
$$

Also the boundary conditions in (2.5) reduces to

$\frac{\partial \psi}{\partial y}=-c x, \frac{\partial \psi}{\partial x}=v_{w}, T=T_{w}, C=C_{w}$

at $\quad y=0$

$\frac{\partial \psi}{\partial y} \rightarrow 0, T \rightarrow T_{\infty}, C \rightarrow C_{\infty}$ as $y \rightarrow \infty$

In order to transform Eqs.(2.10) (2.11) and (2.12) into a set of ordinary differential equations, the following similarity transformations and dimensionless variables are introduced.

$$
\begin{aligned}
& \psi(x, y)=\sqrt{c v} x f(\eta), \quad \eta=\left(\frac{c}{v}\right)^{1 / 2} y, \\
& T=T_{\infty}+\left(T_{w}-T_{\infty}\right) \theta(\eta), \\
& C=C_{\infty}+\left(C_{w}-C_{\infty}\right) \phi(\eta), \quad M=\left(\frac{\sigma B_{0}^{2}}{\rho c}\right)^{1 / 2}, \\
& \operatorname{Pr}=\frac{\mu c p}{k}, S c=\frac{v}{D}, R=\frac{4 \sigma^{*} T_{\infty}^{3}}{K^{\prime} k}, Q=\frac{Q_{0}}{\rho c_{p} c}, \\
& k r^{2}=\frac{k_{0}^{2}}{c}
\end{aligned}
$$

where $f(\eta)$ is the dimensionless stream function, $\theta$ the dimensionless temperature, $\phi$ - the dimensionless concentration, $\eta$ - the similarity variable, $M$ - the magnetic parameter, $P r$ - the Prandtl number, $Q$ - the heat source $(\mathrm{Q}>0)$ or sink $(\mathrm{Q}<0)$ parameter, $S c$ - the Schmidt number and $R$ the radiation parameter.

In view of Eqs.(2.14), the Eqs. (2.10), (2.11) and (2.12) transform into

$$
f^{\prime \prime \prime}+f f^{\prime \prime}-f^{, 2}-M^{2} f^{\prime}=0
$$

$\left(1+\frac{4}{3} R\right) \theta^{\prime \prime}+\operatorname{Pr} f \theta^{\prime}+\operatorname{Pr} Q \theta=0$

$\phi^{\prime \prime}+S c f \phi^{\prime}-k r^{2} \phi=0$

The transformed boundary conditions can be written as

$f=S, f^{\prime}=-1, \theta=1, \phi=1 \quad$ at $\eta=0$

$f^{\prime}=\theta=\phi=0$ as $\eta \rightarrow \infty$

Where $S=v_{w} /(c v)^{1 / 2}(>0)$ is the mass suction parameter.

The main physical quantities of interest are the skin friction coefficient $f$ " $(0)$, the local Nusselt number $-\theta^{\prime}(0)$ and the Sherwood number $-\phi^{\prime}(0)$ which represent the wall shear stress, the heat transfer rate and mass transfer rate at the surface, respectively. Our task is to investigate how the values of $-\theta^{\prime}(0)$ vary with the radiation parameter $R$, magnetic parameter $M$ and Prandtl number $P r$.

\section{SOLUTION OF THE PROBLEM}

The non linear system of Eqs. (2.15) to (2.17) along with the boundary conditions has been solved numerically by finite difference method using the quasilinearization technique.

The discredited version of (2.15)-(2.17) with the boundary conditions (2.18) are written as

$$
\begin{aligned}
& F^{(i+1)}+f^{(i)} F^{(i+1)}-\left(2 F^{(i)}+M^{2}\right) F^{(i+1)}=-F^{2(i)} \\
& \left(1+\frac{4}{3} R\right) \theta^{\prime(i+1)}+\operatorname{Pr} f^{(i+1)} \theta^{\prime(i+1)}+\operatorname{Pr} Q \theta^{(i+1)}=0 \\
& \phi^{\prime(i+1)}+S c f^{(i+1)} \phi^{\prime(i+1)}-S c k r^{2} \phi^{(i+1)}=0 \\
& f^{(i+1)}=S, F^{(i+1)}=-1, \theta^{(i+1)}=1, \phi^{(i+1)}=1 \text { at } \eta=0
\end{aligned}
$$


P. R. Babu et al. / JAFM, Vol. 7, No. 4, pp. 641-650, 2014.

$F^{(i+1)}=\theta^{(i+1)}=\phi^{(i+1)}=0$. as $\eta=\eta^{*}$

The functions with iteration index (i) denote the i-th iteration level and the corresponding index $(i+1)$ be the (i+1)-th level and $\eta^{*}$ is suitable distance from the origin selected by considering the flow behavior in the boundary layer region.

We divide the interval $\left[0, \eta^{*}\right]$ into $\mathrm{N}$ equal subintervals of length $\Delta \eta=0.001$ taking the nondimensional distance $\eta^{*}=50$ for all cases under investigation. Applying central finite difference formulae of the second and first orders derivatives of $\mathrm{F}$ as:

$$
F^{\prime \prime}=\frac{F_{j+1}-2 F_{j}+F_{j-1}}{(\Delta \eta)^{2}} \quad \text { and } F^{\prime}=\frac{F_{j+1}-F_{j-1}}{2(\Delta \eta)}
$$

and similar for $\theta$ and $\phi$, the above system of Eqs. (3.1) to (3.3) along with the boundary conditions (3.4) reduce to

$F_{j-1}^{(i+1)} a_{j}+F_{j}^{(i+1)} b_{j}+F_{j+1}^{(i+1)} c_{j}=d_{j}, 1 \leq j \leq N$

$\theta_{j-1}^{(i+1)} p_{j}+\theta_{j}^{(i+1)} q_{j}+\theta_{j+1}^{(i+1)} r_{j}=0,1 \leq j \leq N$

$\phi_{j-1}^{(i+1)} \alpha_{j}+\phi_{j}^{(i+1)} \beta_{j}+\phi_{j+1}^{(i+1)} \delta_{j}=0,1 \leq j \leq N$

With $f_{0}^{(i+1)}=S, F_{0}^{(i+1)}=-1, \theta_{0}^{(i+1)}=1, \phi_{0}^{(i+1)}=1 \quad$ an $F_{N+1}^{(i+1)}=\theta_{N+1}^{(i+1)}=\phi_{N+1}^{(i+1)}=0$
Where

$$
\begin{aligned}
& a_{j}=\frac{1}{(\Delta \eta)^{2}}-\frac{f^{(i)}}{2(\Delta \eta)}, b_{j}=-\frac{2}{(\Delta \eta)}-2 F_{j}^{(i)}-M^{2} \\
& c_{j}=\frac{1}{(\Delta \eta)^{2}}+\frac{f_{j}^{(i)}}{2(\Delta \eta)}, d_{j}=-F_{j}^{2(i)} \\
& p_{j}=\frac{1}{(\Delta \eta)^{2}}-\left(\frac{3}{3+4 R}\right) \operatorname{Pr} \frac{f_{j}^{(i+1)}}{2(\Delta \eta)} \\
& q_{j}=\frac{-2}{(\Delta \eta)^{2}}+\left(\frac{3}{3+4 R}\right) \operatorname{Pr} Q \\
& r_{j}=\frac{1}{(\Delta \eta)^{2}}+\left(\frac{3}{3+4 R}\right) \operatorname{Pr} \frac{f_{j}^{(i+1)}}{2(\Delta \eta)} \\
& \alpha_{j}=\frac{1}{(\Delta \eta)^{2}}-\frac{S c f_{j}^{(i+1)}}{2(\Delta \eta)}, \beta_{j}=\frac{-2}{(\Delta \eta)^{2}}-S c k r^{2} \\
& \delta_{j}=\frac{1}{(\Delta \eta)^{2}}+\frac{S c f_{j}^{(i+1)}}{2(\Delta \eta)} ; 1 \leq j \leq N
\end{aligned}
$$

We solve the system of algebraic (tri-diagonal system) Eq.(3.5) with the condition (3.8) by the slandered Thomas algorithm. Using the newly obtained results for $f_{j}^{(i+1)}$ and $F_{j}^{(\mathrm{i}+1)}$ the systems (3.6) and (3.7), the discredited temperature and concentration with the conditions (3.8) is them solved by the same thomas algorithm.

\section{RESULTS AND DISCUSSION}

Numerical computations are performed for various values of the physical parameters involved in the equation viz. the Hartmann number (M), the mass suction parameter $(\mathrm{S})$, the Prandtl number $(\mathrm{Pr})$, the radiation parameter, the heat source/sink parameter $(\mathrm{Q})$, The Schmidt number $(\mathrm{Sc})$, chemical reaction parameter (kr). The effects of various parameters on the velocity profiles are depicted in Fig. 1 and 2 . The effects of various parameters on the temperature profiles are depicted in Figs. 3 to 7 . The effects of various parameters on the concentration profiles are depicted in Figs. 8 to 11 .

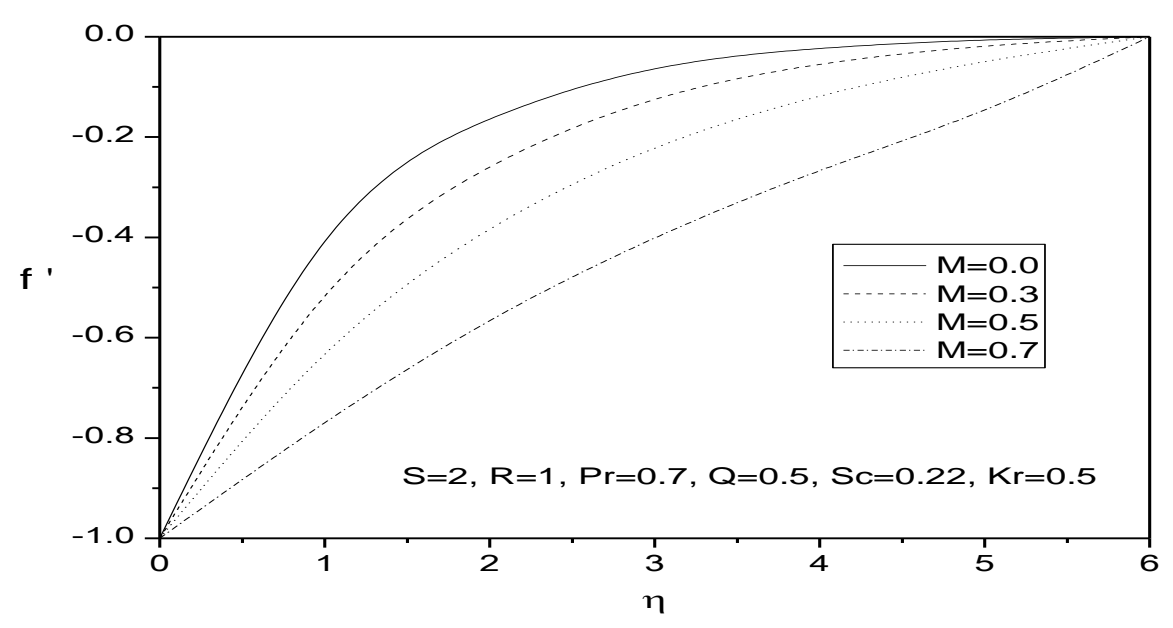

Fig. 1. Velocity profiles for different values of $M$ 
P. R. Babu et al. / JAFM, Vol. 7, No. 4, pp. 641-650, 2014.

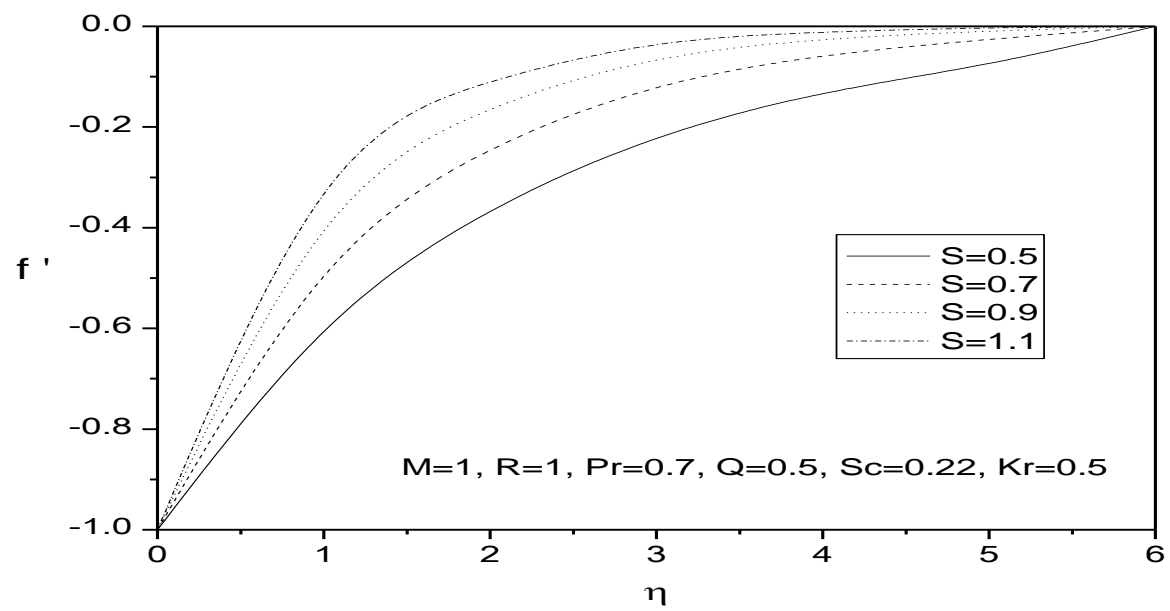

Fig. 2. Velocity profiles for different values of $S$

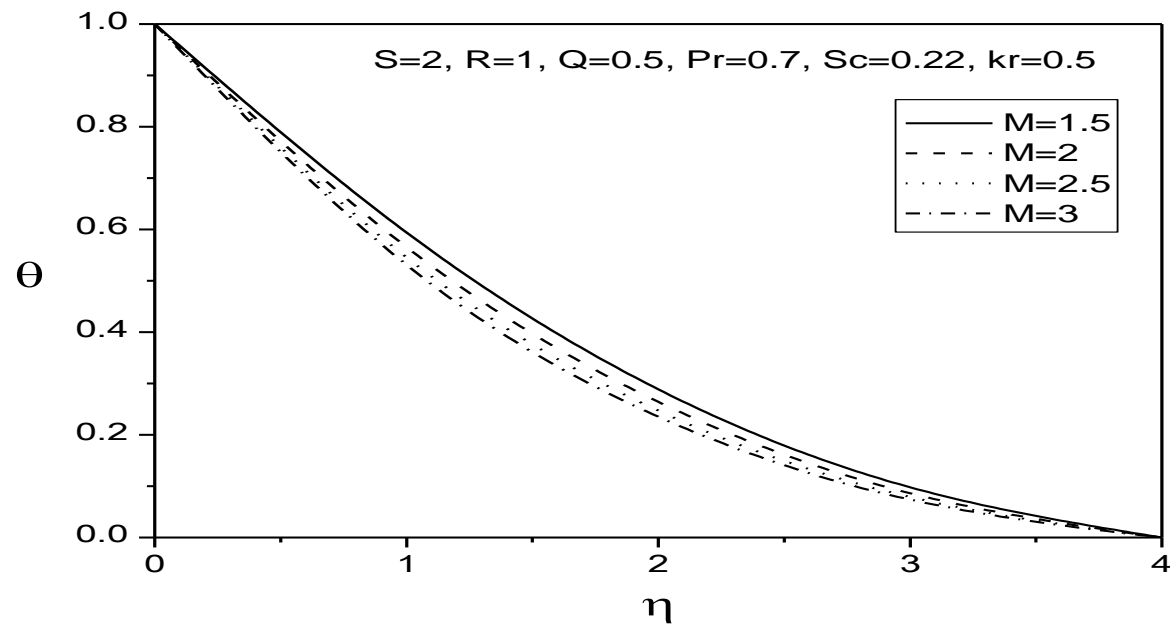

Fig. 3. Temperature profiles for different values of $M$

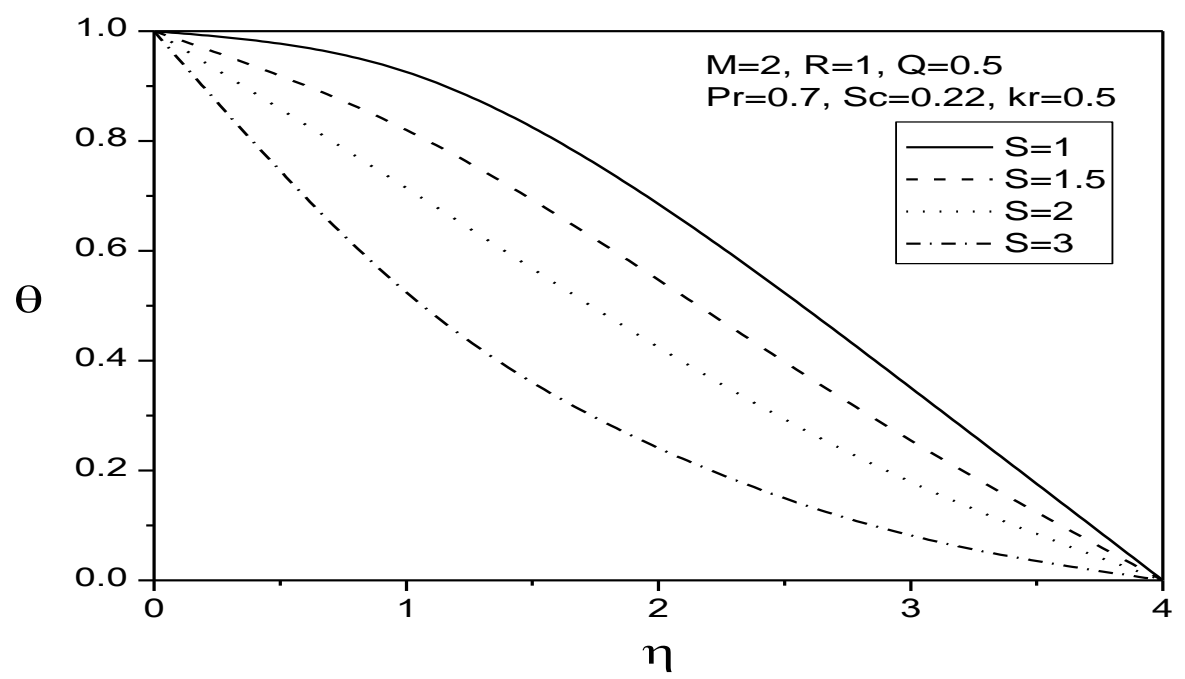

Fig. 4. Temperature profiles for different values of $S$ 
P. R. Babu et al. / JAFM, Vol. 7, No. 4, pp. 641-650, 2014.

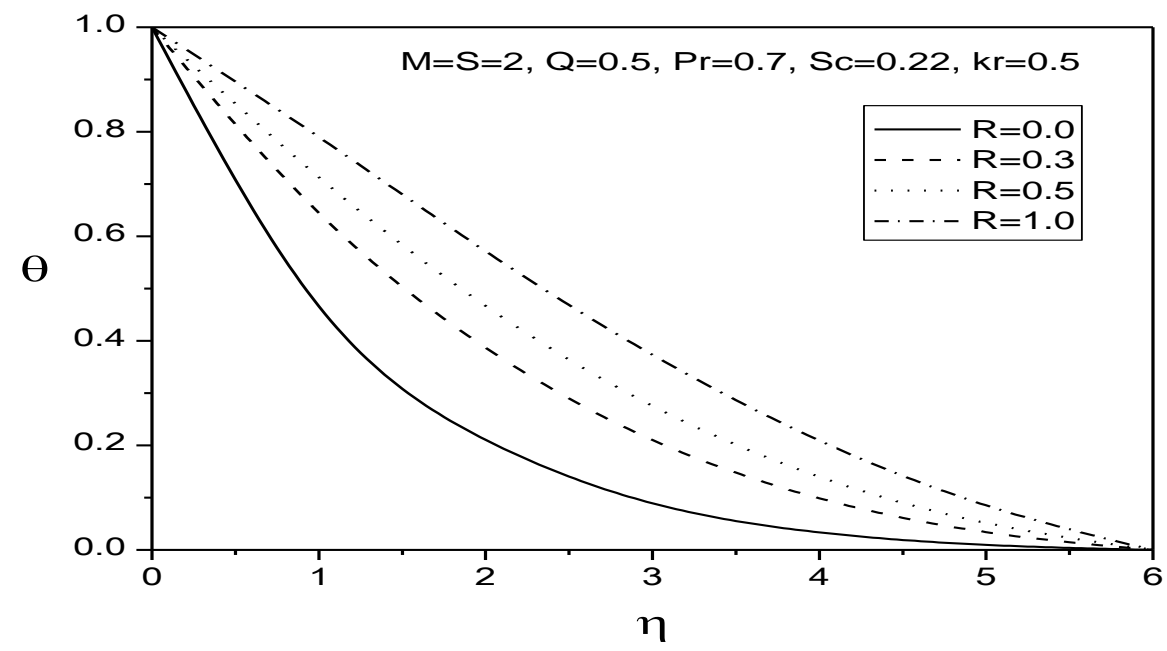

Fig. 5. Temperature profiles for different values of $R$

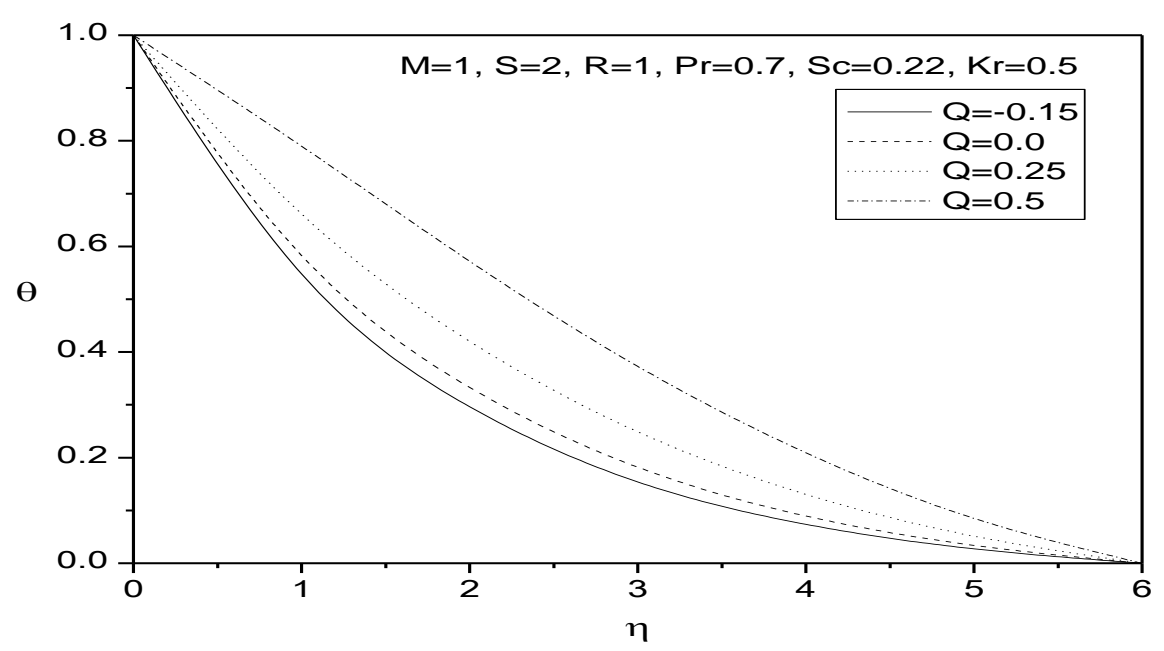

Fig. 6. Temperature profiles for different values of $Q$

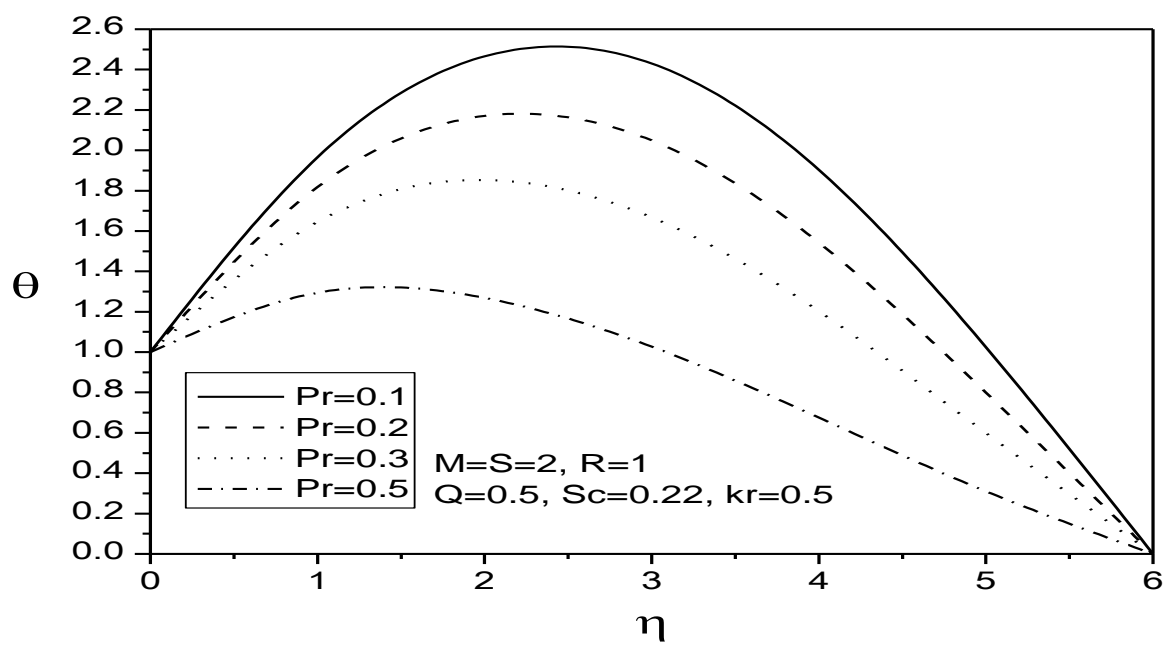

Fig. 7. Temperature profiles for different values of $\mathrm{Pr}$ 
P. R. Babu et al. / JAFM, Vol. 7, No. 4, pp. 641-650, 2014.

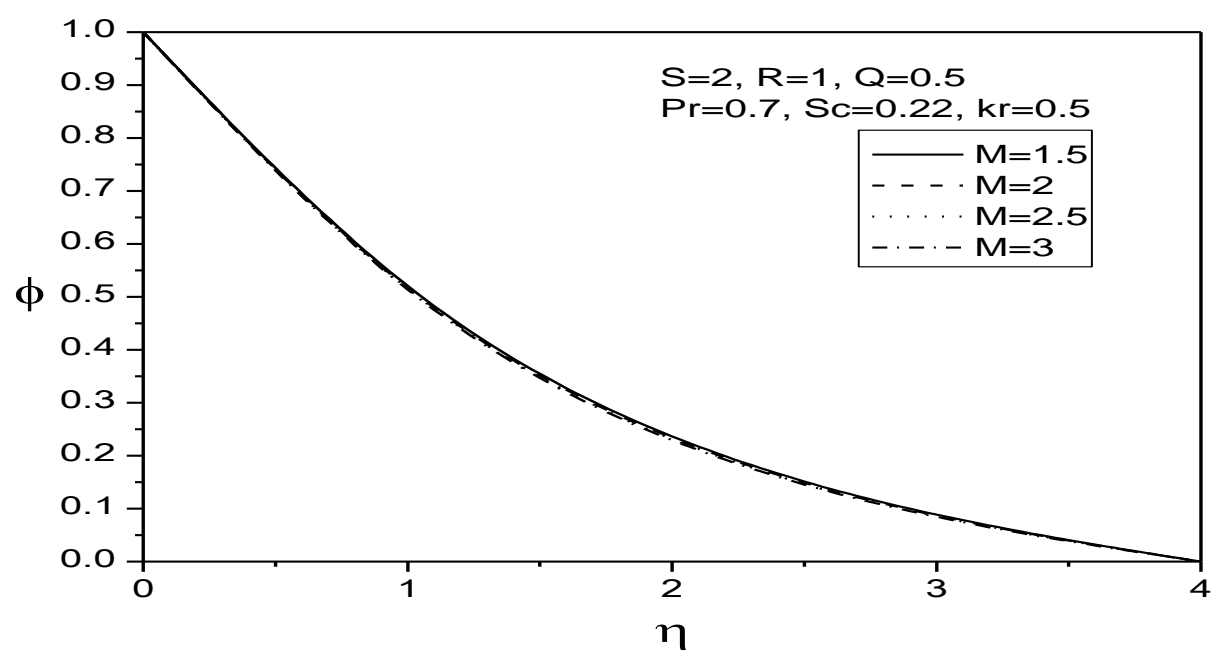

Fig. 8. Concentration profiles for different values of $M$

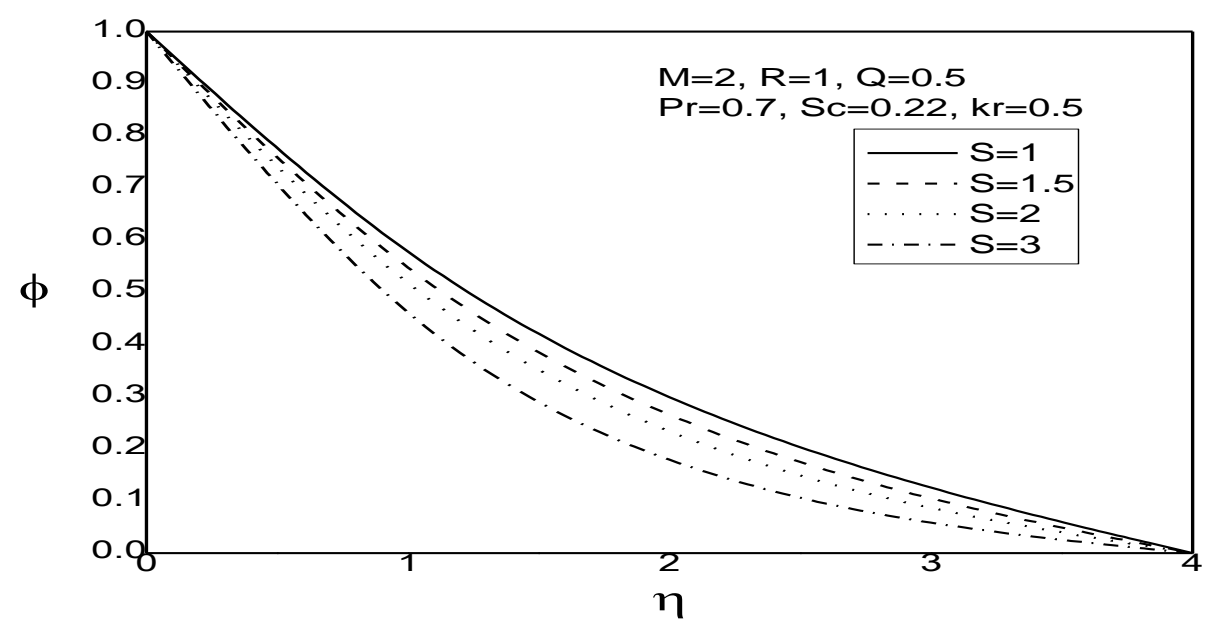

Fig. 9. Concentration profiles for different values of $S$

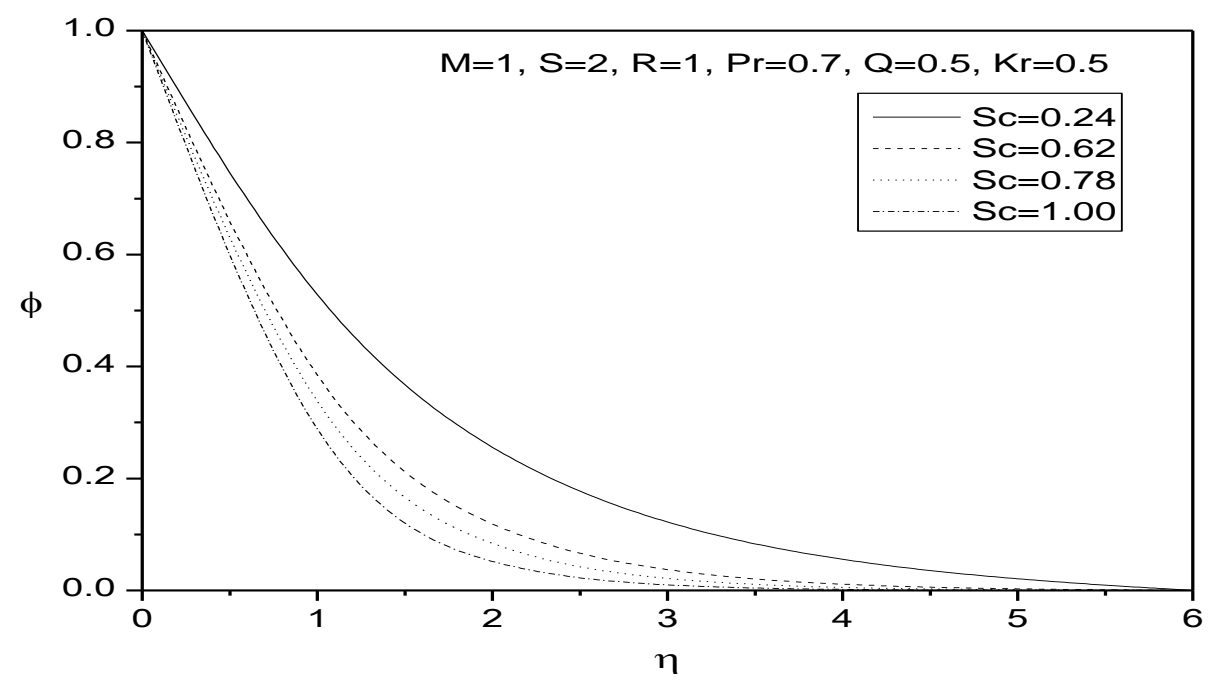

Fig. 10. Concentration profiles for different values of $S c$ 
P. R. Babu et al. / JAFM, Vol. 7, No. 4, pp. 641-650, 2014.

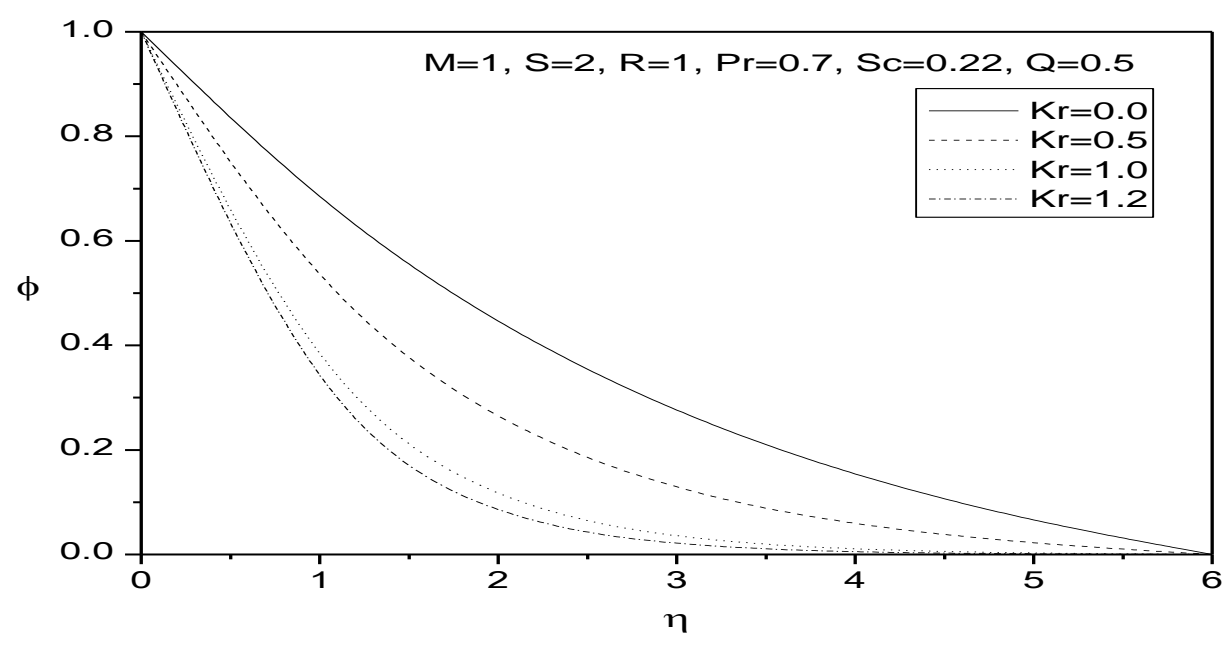

Fig. 11. Concentration profiles for different values of $k r$

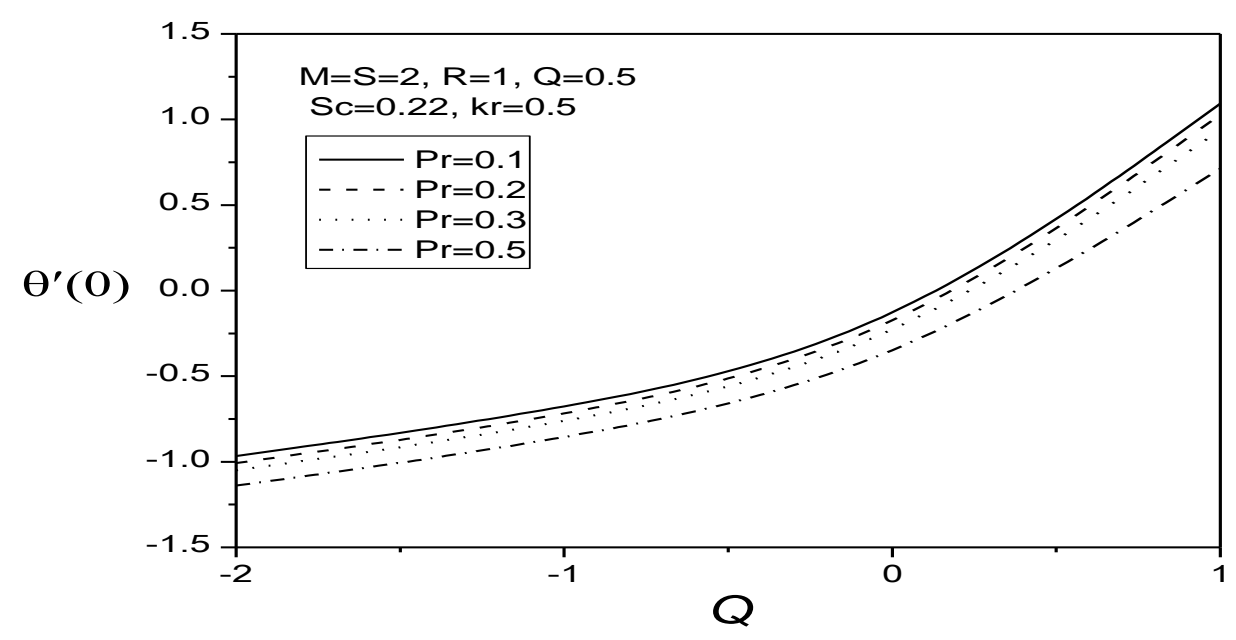

Fig. 12. Variation of the Nusselt number for different $\operatorname{Pr}$ with $Q$

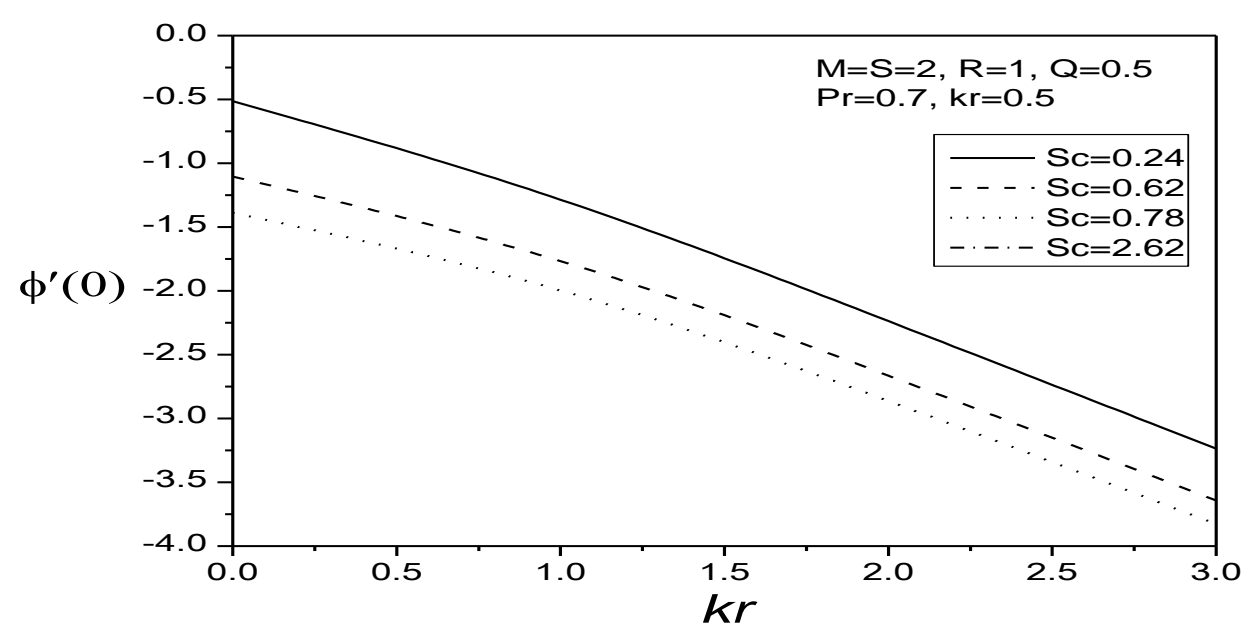

Fig. 13. Variation of the Sherwood number for different $S c$ with $k r$ 
Table 1 Numerical values of $f$ "(0) at the sheet for different values of $\mathbf{S}$ when $M^{2}=2$, Comparison of the present results with that of Bhattacharyya (2011).

\begin{tabular}{|c|c|c|}
\hline S & Bhattacharyya (2011) & Present results \\
\hline 2 & 2.414300 & 2.41448 \\
3 & 3.302750 & 3.30281 \\
4 & 4.236099 & 4.23607 \\
\hline
\end{tabular}

Figure 1 shows the dimensionless velocity profiles for different values in the Hartmann number $(M)$. It is seen that, as expected, the velocity increases with an increase of Hartmann number. Accordingly, the thickness of the momentum boundary layer decreases. This happens due to Lorentz force arising from the interaction of the magnetic and electric fields during the motion of the electrically conducting fluid. To reduce momentum boundary layer thickness the generated Lorentz force enhances the fluid motion in the boundary layer region. The effect of the mass suction parameter on the velocity is illustrated in Fig.2. It is observed that as the mass suction parameter increases, the velocity decreases and it makes the momentum boundary layer thickness thinner.

The effect of the Hartmann number $(M)$ on the temperature is illustrated in Fig.3. It is observed that as the Hartmann number increases, the temperature increases. Fig. 4 depicts the thermal boundary-layer with the mass suction parameter $(S)$. It is noticed that the thermal boundary layer thickness decreases with an increase in the suction parameter. Fig.5 illustrates the effect of the radiation parameter $(R)$ on the temperature. It is noticed that as the radiation parameter increases, the temperature increases. Fig.6 illustrates the effect of the heat source or sink parameter $(Q)$ on the temperature. It is noticed that as the heat source or sink parameter increases, the temperature increases and the lower value of $\mathrm{Q}=-0.25$, high temperature reached . Fig. 7 depicts the temperature profiles with the Prandtl number $(P r)$. It is noticed that the temperature decreases with an increase in the Prandtl number.

The effect of Hartmann number $(M)$ on the concentration field is illustrated Fig.8. As the Hartmann number increases the concentration is found to be decreasing. The effect of mass suction parameter $(S)$ on the concentration field is illustrated Fig. 9. It is noticed that the concentration profiles decreases with an increase in the mass suction parameter. The effect of Schmidt number (Sc) on the concentration field is illustrated Fig. 10. It is noticed that the concentration profiles decreases with an increase in the Schmidt number. Fig. 11 depicts the concentration profiles with the chemical reaction parameter $(k r)$. It is noticed that the concentration decreases with an increase in the chemical reaction parameter.

Figure. 12 shows the variation of the heat transfer rate with the Prandtl number and heat source or sink parameter. It is observed that the heat transfer rate increases with an increase in the Prandtl number, but decreases with an increase in the heat source or sink parameter. Figs. 13 show the variation of the mass transfer rate with the Schmidt number and chemical reaction parameter. It is observed that the mass transfer rate increases with an increase in the Schmidt number and chemical reaction parameter. In Table 1, the present results are compared with those of Bhattacharya [26] and found that there is a perfect agreement.

\section{CONCLUSIONS}

In the present paper, the steady magnetohydrodynamic (MHD) boundary layer flow due to shrinking sheet with mass suction by taking mass transfer and radiation effects into account, are analyzed. The governing equations are approximated to a system of non-linear ordinary differential equations by similarity transformation. The self-similar equations are linearised by the quasilinearization technique and are then by solved by finite difference method. The particular solutions reported in this paper were validated by comparing with solutions existing in the previously published paper. The results are summarized as follows

1. The steady reveals that due to increase of the magnetic field parameter and the mass suction parameter the momentum boundary layer thickness reduces and the temperature decreases.

2. The radiation increases the temperature.

3. The Schmidt number reduces the concentration.

\section{ACNOWLEDGEMENTS}

The author is indebted to the referees for their valuable comments and suggestions, which led to improvement to the paper.

\section{REFERENCES}

Arpaci V. S., (1968), Effect of thermal radiation on the laminar free convection from a heated vertical plate, Int. J. Heat Mass Transfer, 11, 871-881.

Battaler, R. C., (2007), Effects of heat source/sink, Radiation and work done by deformation on flow and heat transfer of a viscoelastic fluid over a stretching sheet, Computers and Mechanics with Applications, 53(2), 305-316.

Brewster, M. Q., (1992), Thermal radiative transfer and properties, John Wiley \& Sons, NewYork.

Callahan, G. D. and Marner, W. J., (1976), Transient free convection with mass transfer on an isothermal vertical flat plate, Int. J .Heat Mass Transfer, 19,165-174. 
Cess R. D. (1966), The interaction of thermal radiation with free convection heat transfer, Int. J.Heat Mass Transfer, 9, 1269-1277.

Chakrabarti, A. and Gupta, A. S., (1979), Hydromagnetic flow and heat trnsfer over a stretching sheet, Quarterly journal of Mechanics and Applied Mechanics, 37, 73-78.

Chen, C. H., (2009), Magneto-hydrodynamic mixed convection of a power-law fluid past a stretching sheer in the presence of thermal radiation and internal heat generation or absorption, international journal of NonLinear Mechanics, 44(6), 596-603.

Crane, L. J., (1970), Flow past a stretching sheet, Zeitschrift fur Angewandte Mathematik and Physik, 21(4),645-647.

Di Clemente, M., Ianiro, A., Rufolo, G., Cardone, G., (2013), Hypersonic test analysis by means of aerothermal coupling methodology and IR thermography, AIAA Journal, 51, 1755-1769.

Elabashbeshy, E. M. A., (1997), Heat and mass transfer along a vertical plate with variable temperature and concentration in the presence of magnetic field, Int. J. Eng. Sci., 34, 515522.

Fang, T., and Zhang, J., (2009), Closed form exact solutions of MHD viscous flow over a shrinking sheet, Communications in Nonlinear science and Numerical solution, 14(7), 28532857.

Gangadhar, K. and Bhaskar Reddy, N., (2013), Chemically Reacting MHD Boundary Layer Flow of Heat and Mass Transfer over a Moving Vertical Plate in a Porous Medium with Suction, Journal of Applied Fluid Mechanics, 6(1), 107-114.

Hayat, T, Abbas, Z., and Sajid, M., (2007), On the analytic solution of magnetohydrodynamic flow of a second grade fluid over a shrinking sheet, Journal of Applied Mechanics, 74, 1165-1171.

Hosaain, M. A. and Takhar, H. S., (1996), Radiation effects on mixed convection along a vertical plate with uniform surface temperature, Heat Mass Transfer, 31, 243-248.

Krishnendu Bhattacharyya, (2011), Effects of heat source/sink on MHD flow and heat transfer over a shrinking sheet with mass suction, Chemical Engineering Reaserch Bulletin, 15, 12-17.
Layek, G. C., (2007), Mukhopadhyay, S., and Samad, S. A., (2007), Heat and mass transfer analysis for boundary layer stagnation point flow towards a heated porous stretching sheet with heat absorption/generation and suction/blowing, international communications in heat and mass transfer, 34(3), 347-356.

Mansour, M. H., (1990), Radiative and free convection effects on the oscillatory flow past a vertical plate, Astrophysics and space science, 166,26-75.

Pavlov. K. B., (1974), Magnetohydrodynamic flow of an incompressible viscous fluid caused by deformation of a plane surface, Magnitnaya Gidrodinamica, 4, 146-147.

Ramachandra Prasad, V., Bhaskar Reddy, N. and Muthucumaraswamy, R., (2007), Radiation and mass transfer effects on two-dimensional flow past an impulsively started isothermal vertical plate, Int. J. Thermal Sciences, 46(12), 1251-1258.

Raptis, A., (1998), Radiation and free convection flow through a porous medium, Int. comm. Heat and Mass Transfer, 25(2), 289-295.

Shanker, B. and Kishan, N., (1997), The effects of mass transfer on the MHD flow past an impulsively started infinite vertical plate with variable temperature or constant heat flux, J. Eng. Heat Mass Transfer, 19, 273-278.

Soundalgekar, V. M., (1979), Effects of mass transfer and free convection currents on the flow past an impulsively started vertical plate, ASME Journal of Applied Mechanics, 46, 757760.

Soundalgekar, V. M. and Wavre, P.D., (1977), Unsteady free convection flow past an infinite vertical plate with constant suction and mass transfer, Int. J. Heat Mass Transfer,20, 13631373.

Wang, C. Y., (1984), The three-dimensional flow due to a stretching flat surface, Physics of Fluids, 27, 1915-1917.

Wang, C. Y., (1990), Liquid film on an unsteady stretching sheet, Quarterly Communications, 48(4), 601-610.

Wang, C. Y., (2008), Stagnation flow towards a shrinking sheet, international journal of NonLinear Mechanics, 43(5), 377-382. 